\title{
COMPARACIÓN DE MÉTODOS DE INTERPOLACIÓN PARA LA GENERACIÓN DE MAPAS DE RUIDO EN ENTORNOS URBANOS
}

\author{
Diego Murillo \\ diego.murillo@usbmed.edu.co
}

\author{
Irene Ortega \\ Universidad de San Buenaventura. Medellín, \\ ireop_5@hotmail.com \\ Juan David Carrillo
Buenaventura. Medellín, \\ juandc0389@gmail.com
}

Andrés Pardo

Colombia

flppardo@gmail.com Jeiser80@gmail.com

(Tipo de Artículo: Investigación. Recibido el 07/02/2012. Aprobado el 08/05/2012)

\section{RESUMEN}

En este trabajo se presenta el estudio efectuado con el propósito de analizar la congruencia de los métodos de interpolación en la generación de mapas de ruido. Para esto, se realizaron mediciones del nivel de presión sonora equivalente de acuerdo con la resolución 0627 del Ministerio del Medio Ambiente, Vivienda y Desarrollo Territorial en dos áreas de la ciudad de Medellín. Para la obtención de las curvas de niveles de ruido se utilizaron los métodos de interpolación Kriging e IDW en el software SIG ArcGis. En el aplicativo se varió la configuración de los parámetros de entrada y se efectuó un análisis estadístico para determinar qué modelo presentaba resultados más precisos. Por último, se llevó a cabo una valoración del mapa de ruido obtenido comparándolo con la legislación colombiana.

Palabras clave

Interpolación, SIG, mapas de ruido, ruido ambiental.

\section{A COMPARISON OF INTERPOLATION METHODS FOR CREATING NOISE MAPS IN URBAN ENVIRONMENTS}

\begin{abstract}
This paper presents a study focused on the analysis of the congruence of interpolation methods for creating noise maps. For this purpose noise measurements were performed in two areas of Medellin - Colombia- in compliance with the National Standard 0627 issued in 2006. Subsequently, this information was analyzed in the software for geographic information systems called ArcGIS. To obtain the noise curves the Kriging and IDW interpolation methods were used, varying the configuration of the input parameters and performing a statistical analysis to determine which model had more accurate results. Finally, an assessment was made by comparing the resultant noise map with the issues established by Colombian laws.
\end{abstract}

\section{Keywords}

Environmental noise, Geographic Information Systems (GIS), interpolation, noise mapping.

\section{UNE COMPARAISON DES METHODES D'INTERPOLATION POUR LA CRÉATION DES CARTES DE BRUIT DANS ENVIRONNEMENTS URBAINES}

\section{RÉSUMÉ}

Cet article présent une étude réalisée avec l'intention d'analyser la congruence des méthodes d'interpolation dans la création des cartes de bruit. Pour réussir cela, on a réalisé des mesures des niveaux de bruit d'après la résolution 0627 du Ministère d'Environnement, Logement et Développement Territorial dans deux zones de la ville de Medellín-Colombie. Pour trouver les courbes de niveau de bruit on a utilisée les méthodes d'interpolation Kriging et IDW sur le logiciel pour systèmes d'information géographique appelé ArcGIS. Dans l'application on a varié la configuration des paramètres d'entrée et on a réalisé une analyse statistique pour déterminer quel modèle présentait des résultats plus précis. Finalement, on a fait une évaluation de la carte de bruit obtenu au moyen d'une comparaison avec ce qu'établit la législation colombienne.

\section{Mots-clés}

Interpolation, Systèmes d'Information Géographique (SIG), Cartes de bruit, Bruit de l'environnement. 


\section{INTRODUCCIÓN}

Los mapas de ruido se han convertido en las últimas décadas en un instrumento esencial para la caracterización del grado de contaminación acústica en las ciudades y la generación de planes de mitigación sonora. La información obtenida de los mismos, permite a los entes gubernamentales tomar decisiones con relación a la planificación del territorio, estableciendo niveles máximos de acuerdo a los usos del suelo que en la ciudad existan. En Colombia, la Resolución 0627 de 2006 estipula la metodología para la medición de los niveles de ruido ambiental así como los estándares máximos permisibles de acuerdo al sector y las actividades que en él se desarrollan. Igualmente resuelve que estas mediciones deben ser insumo para la elaboración de mapas de ruido en las ciudades que posean más de 100.000 habitantes [1].

Para la elaboración de los mapas existen diferentes metodologías que permiten la construcción de las curvas de ruido. Usualmente, se realizan mediciones in situ de ruido ambiental que posteriormente, al aplicar técnicas de interpolación, se estiman valores desconocidos a partir de los registros realizados. Actualmente los métodos más usados son Kriging e IDW (Inverse Distance Weighting) que se basan en la auto-correlación espacial de los puntos para la predicción y generación de superficies continuas.

Estas técnicas de interpolación han sido ampliamente utilizadas para la generación mapas de ruido ya que permiten crear superficies continuas facilitando el análisis de la condición acústica del sector en estudio [2 - 5]. Amal laaly-Sankary et al. [6] elaboraron el mapa de ruido de la ciudad El-Mina mediante una rejilla de $50 \mathrm{~m}$ para sectores densamente poblados y de $150 \mathrm{~m}$ para los menos poblados; la técnica de interpolación utilizada fue IDW y como conclusión se pudo determinar los sectores con mayor grado de contaminación sonora [6]. Surya Aditya et al. [7] realizaron el mapa de ruido de Rawatpur combinando mediciones en campo con modelado por medio de software. Para ello realizaron 56 mediciones durante intervalos de tiempo en los que existieran altos niveles emisión de ruido automotor. Cada uno de los puntos fue geo-referenciado y con un SIG se realizó el proceso de interpolación por medio de Kriging [7].

En el contexto nacional el área metropolitana del Valle de Aburrá realizó el mapa de ruido de Medellín, Bello, La Estrella, Sabaneta, Itagüí, Girardota, Copacabana, Barbosa y Caldas utilizando el método de interpolación Kriging. En este estudio se seleccionaron diferentes tamaños de rejilla con base a la zona bajo estudio; en municipios como La Estrella se realizó el estudio con una cuadrícula de 200 metros para un total de 77 puntos, mientras que para el mapa de ruido de Medellín se utilizó una de 400 metros para un total de 418 puntos [8].

\section{BASES TEÓRICAS}

En estadística la interpolación es usada para estimar valores desconocidos a partir de una serie de datos conocidos y ponderados. Cada técnica busca determinar una función que represente adecuadamente el fenómeno bajo estudio y así disminuir la cantidad de información a recolectar en campo. Existen varias técnicas o métodos, entre los que se encuentran IDW y Kriging.

\subsection{Inverse Distance Weighting (IDW)}

Es un método matemático de interpolación que usa una función inversa de la distancia, parte del supuesto que las cosas que están más cerca son más parecidas, por lo tanto tienen más peso e influencia sobre el punto a estimar [9]. Matemáticamente se expresa como:

$$
Z\left(S_{o}\right)=\sum_{i=1}^{N} \lambda_{i} * Z\left(S_{i}\right)
$$

En el cual $Z\left(S_{0}\right)$ es el valor a predecir, $N$ es el número de muestras alrededor del punto a predecir, $\lambda_{i}$ son los pesos asignados a cada punto vecino y $Z\left(S_{i}\right)$ son los valores medidos. Los pesos de los puntos vecinos están dados por:

$$
\lambda_{i}=\frac{d_{i o}-p}{\sum_{i=1}^{N} d_{i o}{ }^{-p}}
$$

En el cual $d$ es la distancia entre el lugar de predicción $\left(S_{o}\right)$ y el lugar muestral $\left(S_{i}\right)$; $P$ es un factor de reducción de peso, cuyo valor se encuentra minimizando el error cuadrático medio o error de predicción.

\subsection{Kriging ordinario}

Es un método basado en auto correlación espacial de las variables. El Kriging es un estimador lineal insesgado que busca generar superficies continuas a partir de puntos discretos. Asume que la media, aunque desconocida, es constante y que las variables son estacionarias y no tienen tendencias. Permite transformación de los datos, eliminación de tendencias y proporciona medidas de error.

Para determinar la auto correlación se usan semivariogramas y vecindades obtenidos a partir de un análisis exploratorio de los datos. El semivariograma permite a partir de la covarianza entre los puntos, representar la variabilidad de los mismos y su dependencia en función de la distancia y la dirección. La fórmula general es:

$$
Z(S)=\mu(S)+\varepsilon(S)
$$

En el cual $Z(S)$ es la variable de interés, $\mu(S)$ es una constante desconocida, $\varepsilon(S)$ son errores aleatorios de estimación y $S$ son coordenadas espaciales $(x, y)$. La predicción de un punto está dada por:

$$
\hat{Z}\left(S_{o}\right)=\sum_{i=1}^{N} \lambda_{i} Z\left(S_{i}\right)
$$

En el cual $\hat{Z}\left(S_{o}\right)$ es el valor predicho, $\lambda_{i}$ es el peso de cada valor observado y $Z\left(S_{i}\right)$ es el valor medido en un lugar. Además:

$$
\varepsilon=Z\left(S_{o}\right)-\sum \lambda_{i} Z\left(S_{i}\right)
$$


Para que los valores pronosticados no estén sesgados la suma de los pesos debe ser igual a 1. Para minimizar el error se agrega el multiplicador de LaGrange $(\Pi)$, obteniendo:

$$
\Gamma * \lambda=g
$$

En el cual $\Gamma$ es la matriz de valores del semivariograma teórico, $\lambda$ es el vector de pesos a encontrar y $g$ es el vector de semivarianza teórica en cada punto.

\section{MÉTODOS}

El barrio Laureles es un sector residencial en el cual existen algunos establecimientos comerciales y cuya fuente principal de ruido es el tráfico automotor. Se caracteriza por ser una zona tranquila en el interior del barrio pero con altos niveles de ruido en las periferias debido a las vías principales. San Benito es un sector comercial en el que se encuentran inmersas varias instituciones educativas además de contener pequeña y mediana industria. La principal fuente de ruido es el tráfico automotor, teniendo mayor incidencia en las vías perimetrales de la zona de estudio.

Para el desarrollo del mapa en Laureles se efectuaron 48 mediciones en un área aproximada de 517.447,08 $\mathrm{m}^{2}$ (Figura 1). En San Benito se registraron 40 mediciones en un área aproximada de 139.609,83 $\mathrm{m}^{2}$ (Figura 2). El procedimiento de medición se realizó con base en la resolución 0627 de 2006.

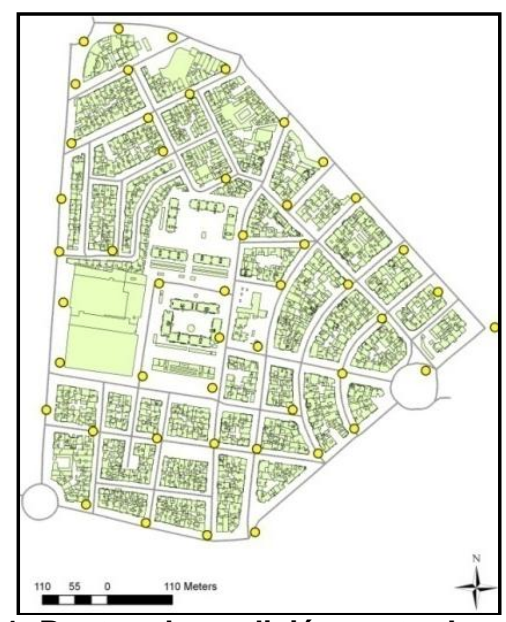

Fig. 1: Puntos de medición sector Laureles

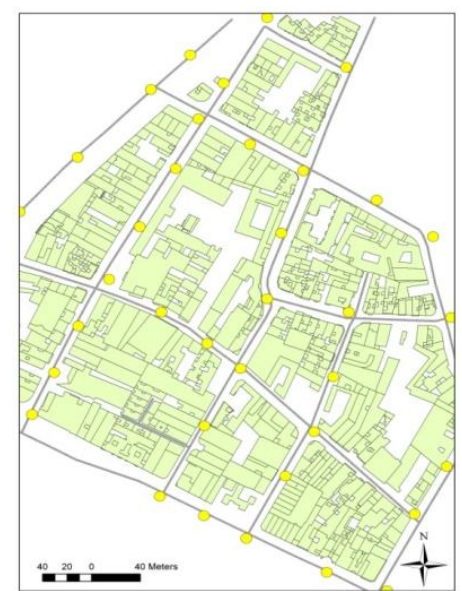

Fig. 2: Puntos de medición sector San Benito
Luego de las mediciones se procedió a la construcción de las curvas de ruido en el software ArcGis. El primer paso consistió en un análisis exploratorio con el fin de identificar sesgos en las mediciones y tendencias en los datos. Para esto, se corroboró que la información adquirida se ajustara a una distribución normal y así determinar si era necesario efectuar una transformación en los datos. Como se evidencia en las Figuras 3 y 4, los datos se ajustan a una distribución normal con algunas excepciones que corresponden a puntos específicos en los que los niveles de ruido presentan una variación debido a calles cerradas o glorietas en donde el flujo variaba significativamente en el intervalo de medición.

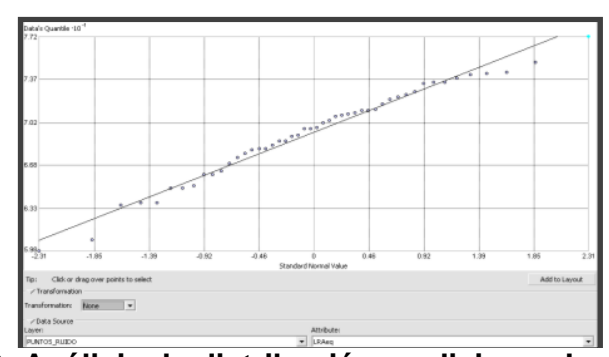

Fig. 3: Análisis de distribución mediciones Laureles

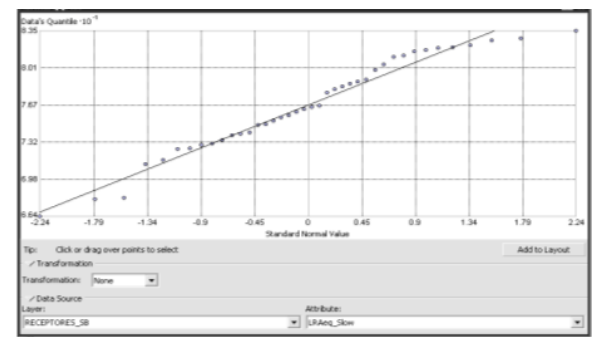

Fig. 4: Análisis de distribución mediciones San Benito

Realizado este proceso, se efectuó un análisis de tendencias identificando concentraciones en la información. En las Figuras $5 a$ a $5 c$ y $6 a$ a $6 c$ se presentan las tendencias de acuerdo a un polinomio de grado uno, dos y tres. En los mismos se puede observar que existe una favorabilidad hacia la dirección occidente-oriente y sur-norte en el sector de Laureles y oriente-occidente en el caso de San Benito.

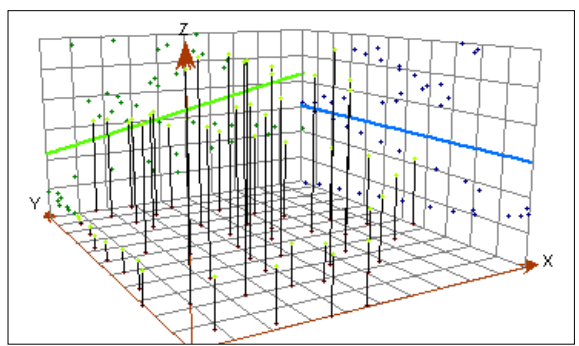

Fig. 5a: Análisis tendencias Laureles: polinomio grado 1

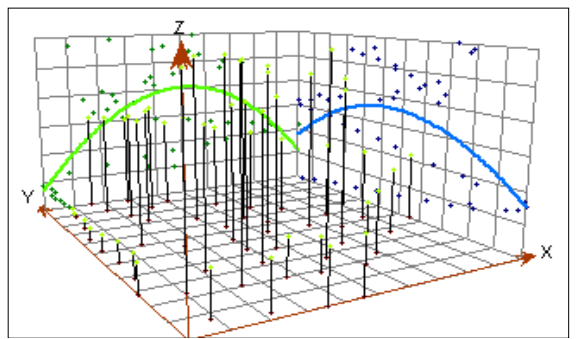

Fig. 5b: Análisis tendencias Laureles: polinomio grado 2 


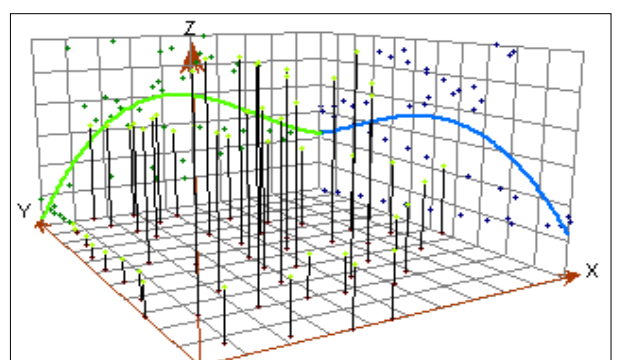

Fig. 5c: Análisis tendencias Laureles: polinomio grado 3

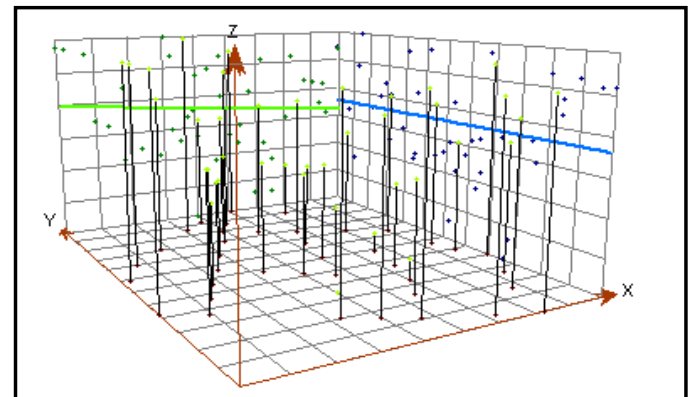

Fig. 6a: Análisis San Benito: polinomio grado 1

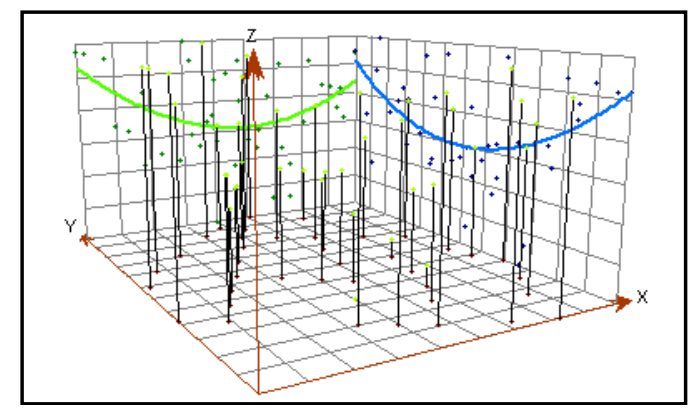

Fig. 6b: Análisis San Benito: polinomio grado 2

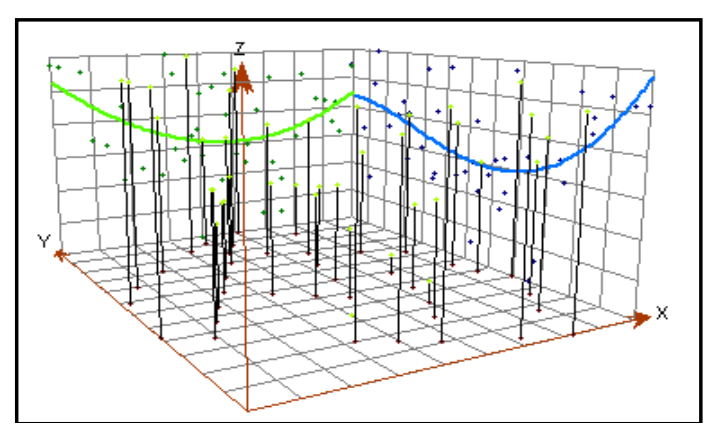

Fig. 6c: Análisis San Benito: polinomio grado 3

Una vez efectuado el análisis exploratorio se generaron múltiples escenarios (15 por sector) con el método Kriging e IDW en los que se varió el factor de potencia, la forma y ángulo de vecindad, el número de vecinos y el modelo teórico del semivariograma. Como criterio de selección se determinó que la media del error, la varianza, el error promedio estándar, la media estandarizada y la varianza estandarizada fueran lo menor posible. Igualmente se predijeron los puntos medidos y se analizó cual escenario tuvo mayor congruencia entre los estadísticos (media, máximo, mínimo, suma y desviación estándar) de los datos predichos y los medidos [9].

\section{RESULTADOS}

En las Figuras $7 a$ a $7 d$ se presentan los mapas generados con el método Kriging para cada sector.

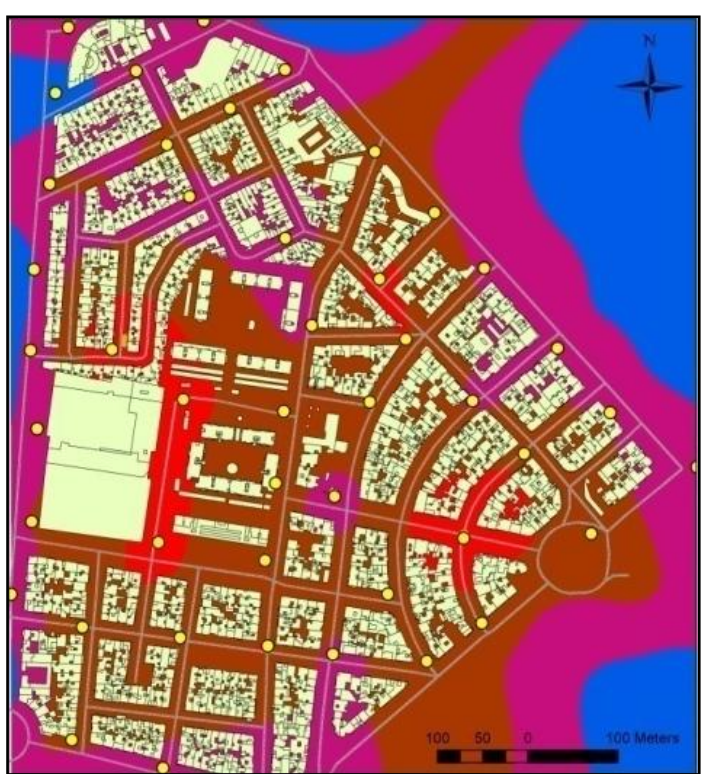

Fig. 7a: Modelo 1 método Kriging sector Laureles

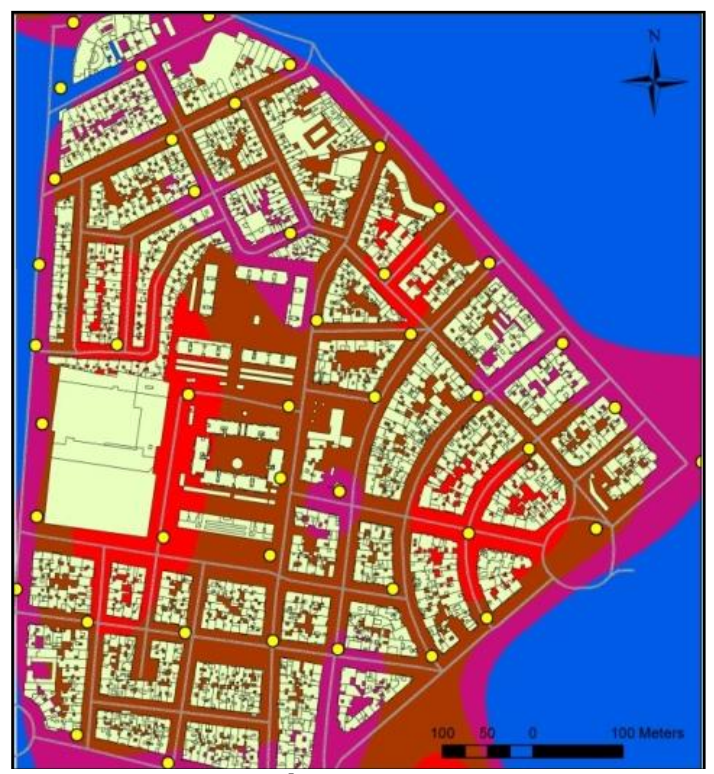

Fig. 7b: Modelo 2 método Kriging sector Laureles

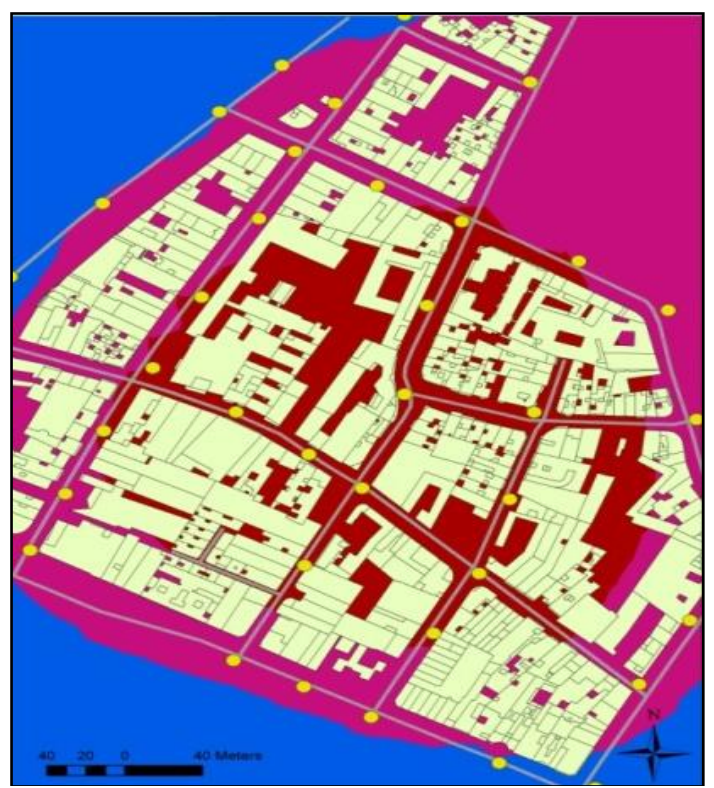

Fig. 7c: Modelo 1 método Kriging sector San Benito 


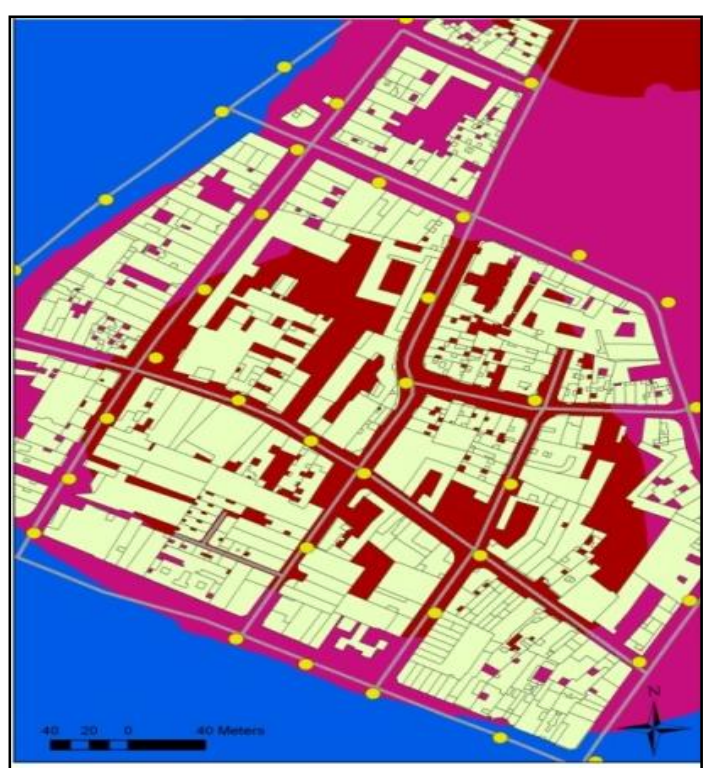

Fig. 7d: Modelo 2 método Kriging sector San Benito

En las Tablas 1 a 4 se muestran los parámetros seleccionados para determinar la congruencia del proceso de interpolación en ambos sectores. Como base se analizan los estadísticos: media, mínimo, máximo, desviación estándar, obtenidos con los valores registrados en campo y los resultantes por medio de la predicción con el método de interpolación.

TABLA 1. Modelo 1 Laureles

\begin{tabular}{|c|c|c|c|c|c|}
\hline $\begin{array}{l}\text { TEINDENCIA } \\
\text { REMOVIDA }\end{array}$ & MODELC & \multicolumn{2}{|c|}{ ESTADISTICOS } & & $\begin{array}{c}\text { RAINGODE } \\
\text { ERROR }\end{array}$ \\
\hline \multirow[t]{5}{*}{1} & ESFERICC & \multicolumn{2}{|c|}{ Media } & 0.4899 & -5.894 \\
\hline & & \multicolumn{2}{|c|}{ RMS } & 3.298 & 8.3067 \\
\hline & & \multicolumn{2}{|c|}{ Error Promedio Estandar } & 0.115 & \\
\hline & & \multicolumn{2}{|c|}{ Media Estandarizada } & 4.284 & \\
\hline & & \multicolumn{2}{|c|}{ RMS Estandarizado } & 28.72 & \\
\hline \multicolumn{3}{|c|}{ PREDICHOS } & \multicolumn{2}{|c|}{ MEDIDOS } & DIFERENCIA \\
\hline \multicolumn{2}{|c|}{ Minimo } & 64.637556 & \multicolumn{2}{|c|}{59.8} & 4.837556 \\
\hline \multicolumn{2}{|c|}{ Maximo } & 76.225917 & \multicolumn{2}{|c|}{77.2} & -0.974083 \\
\hline \multicolumn{2}{|c|}{ Suma } & 3355.714873 & \multicolumn{2}{|c|}{3332.2} & 23.514873 \\
\hline \multicolumn{2}{|c|}{ Media } & 69.910727 & \multicolumn{2}{|c|}{69.420833} & 0.489894 \\
\hline \multicolumn{2}{|c|}{ Desviacion Estandar } & 2.594176 & \multicolumn{2}{|c|}{3.745551} & -1.151375 \\
\hline
\end{tabular}

TABLA 2. Modelo 2 Laureles

\begin{tabular}{|c|c|c|c|c|c|}
\hline $\begin{array}{l}\text { TENDENCIA } \\
\text { REMOVIDA }\end{array}$ & MODELO & \multicolumn{2}{|c|}{ ESTADISTICOS } & & $\begin{array}{c}\text { RANGO DE } \\
\text { ERROR }\end{array}$ \\
\hline \multirow[t]{5}{*}{3} & ESFERICC & \multicolumn{2}{|c|}{ Media } & -0.079 & -5.8961 \\
\hline & & \multicolumn{2}{|c|}{ RMS } & 3.056 & 6.8114 \\
\hline & & \multicolumn{2}{|c|}{ Error Promedio Estandar } & 0.1968 & \\
\hline & & \multicolumn{2}{|c|}{ Media Estandarizada } & -0.406 & \\
\hline & & \multicolumn{2}{|c|}{ RMS Estandarizado } & 15.54 & \\
\hline \multicolumn{3}{|c|}{ PREDICHOS } & \multicolumn{2}{|c|}{ MEDIDOS } & DIFERENCIA \\
\hline \multicolumn{2}{|c|}{ Minimo } & 61.453422 & \multicolumn{2}{|c|}{59.8} & 1.653422 \\
\hline \multicolumn{2}{|c|}{ Maximo } & 76.921319 & \multicolumn{2}{|c|}{77.2} & -0.278681 \\
\hline \multicolumn{2}{|c|}{ Suma } & 3328.390838 & \multicolumn{2}{|c|}{3332.2} & -3.809162 \\
\hline \multicolumn{2}{|c|}{ Media } & 69.341476 & \multicolumn{2}{|c|}{69.420833} & -0.079357 \\
\hline \multicolumn{2}{|c|}{ Desviacion Estandar } & 3.324884 & \multicolumn{2}{|c|}{3.745551} & -0.420667 \\
\hline
\end{tabular}

TABLA 3. Modelo 1 San Benito

\begin{tabular}{|c|c|c|c|c|}
\hline $\begin{array}{c}\text { TENDENCIA } \\
\text { REMOVIDA }\end{array}$ & MODELO & ESTADISTICOS & & $\begin{array}{c}\text { RANGO DE } \\
\text { ERROR }\end{array}$ \\
\hline 2 & ESFERICO & Media & 0.0003 & -4.582 \\
\hline & & RMS & 3.46 & 10.446 \\
\hline & & Error Promedio Estandar & 2.443 & \\
\hline & & Media Estandarizada & 0.0009 & 15.028 \\
\hline & & RMS Estandarizado & 1.415 & \\
\hline
\end{tabular}

\begin{tabular}{|c|c|c|c|}
\hline \multicolumn{2}{|c|}{ PREDICHOS } & MEDIDOS & DIFERENCIA \\
\hline Minimo & 71.243374 & 66.4 & 4.843374 \\
\hline Maximo & 83.183786 & 83.5 & -0.316214 \\
\hline Suma & 3065.210654 & 3065.2 & 0.010654 \\
\hline Media & 76.630266 & 76.63 & 0.000266 \\
\hline Desviacion Estandar & 3.138516 & 4.335447 & -1.196931 \\
\hline
\end{tabular}

TABLA 4. Modelo 2 San Benito

\begin{tabular}{|c|c|c|c|c|c|}
\hline $\begin{array}{l}\text { TENDENCIA } \\
\text { REMOVIDA }\end{array}$ & MODELO & \multicolumn{2}{|c|}{ ESTADISTICOS } & & $\begin{array}{l}\text { RANGO DE } \\
\text { ERROR }\end{array}$ \\
\hline \multirow[t]{5}{*}{3} & ESFERICO & \multicolumn{2}{|c|}{ Media } & -0.004 & -9.5354 \\
\hline & & \multicolumn{2}{|c|}{ RMS } & 3.692 & 9.2471 \\
\hline & & \multicolumn{2}{|c|}{ Error Promedio Estandar } & 2.576 & \\
\hline & & \multicolumn{2}{|c|}{ Media Estandarizada } & -0.003 & 18.7825 \\
\hline & & \multicolumn{2}{|c|}{ RMS Estandarizado } & 1.428 & \\
\hline \multicolumn{3}{|c|}{ PREDICHOS } & \multicolumn{2}{|c|}{ MEDIDOS } & DIFERENCIA \\
\hline \multicolumn{2}{|c|}{ Minimo } & 70.139214 & \multicolumn{2}{|c|}{66.4} & 3.739214 \\
\hline \multicolumn{2}{|c|}{ Maximo } & 83.97969 & \multicolumn{2}{|c|}{83.5} & 0.47969 \\
\hline \multicolumn{2}{|c|}{ Suma } & 3065.028922 & \multicolumn{2}{|c|}{3065.2} & -0.171078 \\
\hline \multicolumn{2}{|c|}{ Media } & 76.625723 & \multicolumn{2}{|c|}{76.63} & -0.004277 \\
\hline \multicolumn{2}{|c|}{ Desviacion Estandar } & 3.61144 & \multicolumn{2}{|c|}{4.335447} & -0.724007 \\
\hline
\end{tabular}

En las Figuras $8 \mathrm{a}$ a $8 \mathrm{~d}$ se presentan los mapas generados por el método IDW para cada sector.

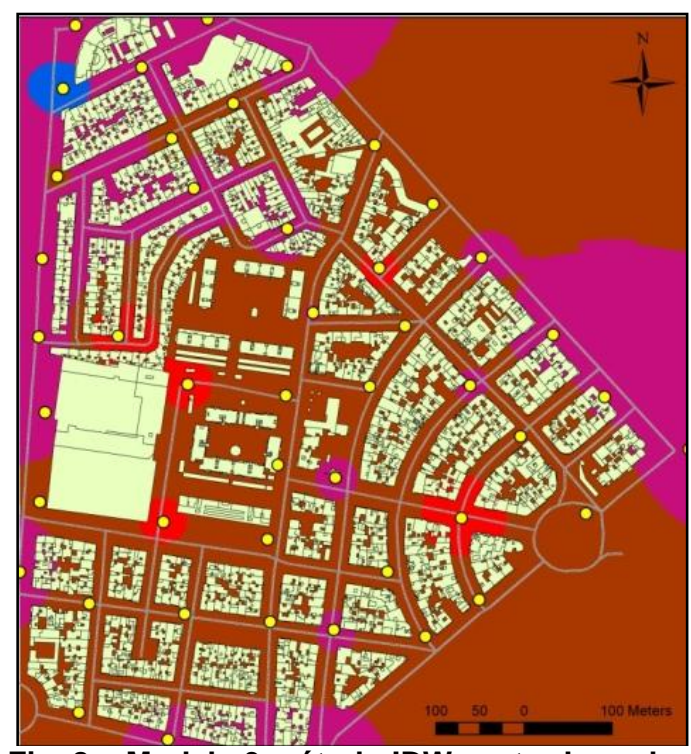

Fig. 8a: Modelo 3 método IDW sector Laureles

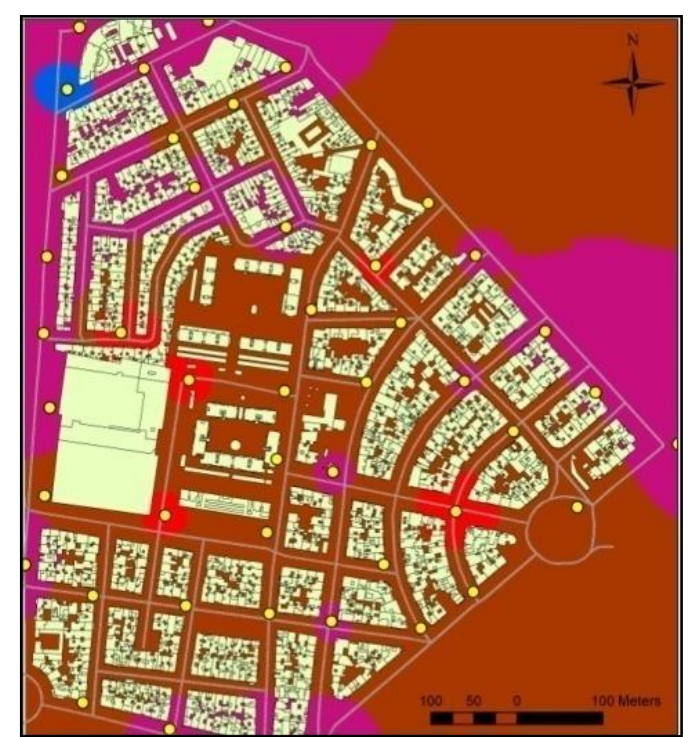

Fig. 8b: Modelo 4 método IDW sector Laureles 


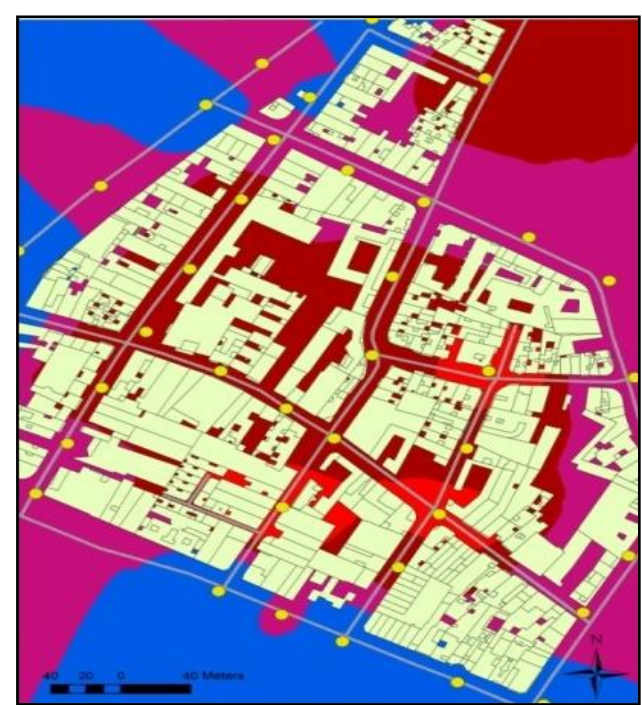

Fig. 8c: Modelo 3 método IDW sector San Benito

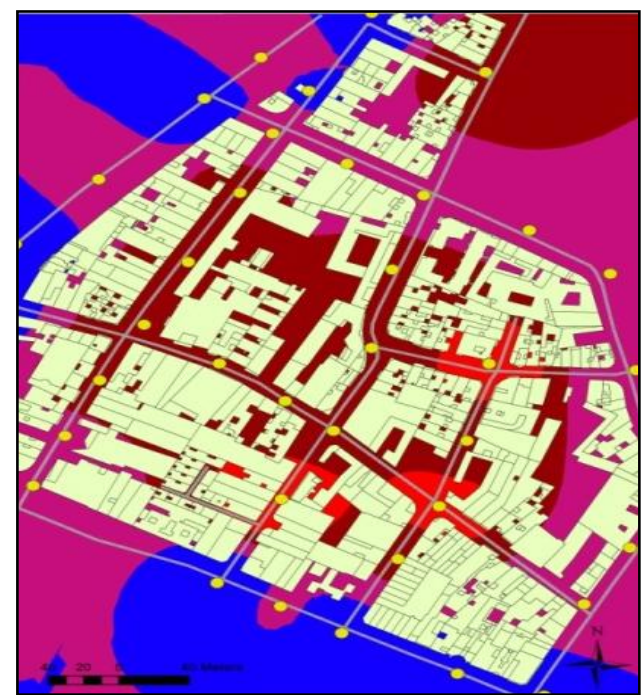

Fig. 8d: Modelo 4 método IDW sector San Benito

El análisis de estadísticos resultantes del método IDW en ambos sectores se muestra en la Tablas 5 a 8.

TABLA 5. Modelo 3 Sector Laureles

\begin{tabular}{|c|c|c|c|c|c|}
\hline \multicolumn{2}{|c|}{ PROPIEDADES } & \multicolumn{2}{|c|}{ ESTADISTICOS } & & $\begin{array}{c}\text { RANGO DE } \\
\text { ERROR }\end{array}$ \\
\hline $\mathrm{x}$ & 286 & \multicolumn{2}{|c|}{ Media } & -0.128 & -6.4622 \\
\hline $\mathrm{Y}$ & 286 & \multicolumn{2}{|c|}{ RMS } & 3.459 & 9.4739 \\
\hline Angulo & 333 & & & & \\
\hline \multicolumn{3}{|c|}{ PREDICHOS } & \multicolumn{2}{|c|}{ MEDIDOS } & DIFERENCIA \\
\hline \multicolumn{2}{|c|}{ Minimo } & 66.267117 & \multicolumn{2}{|c|}{59.8} & 6.467117 \\
\hline \multicolumn{2}{|c|}{ Maximo } & 74.776605 & \multicolumn{2}{|c|}{77.2} & -2.423395 \\
\hline \multicolumn{2}{|c|}{ Suma } & 3326.081026 & \multicolumn{2}{|c|}{3332.2} & -6.118974 \\
\hline \multicolumn{2}{|c|}{ Media } & 69.293355 & \multicolumn{2}{|c|}{69.420833} & -0.127478 \\
\hline \multicolumn{2}{|c|}{ Desviacion Estandar } & 2.000611 & \multicolumn{2}{|c|}{3.745551} & -1.74494 \\
\hline
\end{tabular}

TABLA 6. Modelo 4 Sector Laureles

\begin{tabular}{|c|c|c|c|c|c|}
\hline \multicolumn{2}{|c|}{ PROPIEDADES } & \multicolumn{2}{|c|}{ ESTADISTICOS } & & $\begin{array}{l}\text { RANGO DE } \\
\text { ERROR }\end{array}$ \\
\hline$x$ & 286 & \multicolumn{2}{|c|}{ Media } & -0.081 & -6.3584 \\
\hline $\mathrm{Y}$ & 286 & \multicolumn{2}{|c|}{ RMS } & 3.397 & 8.7366 \\
\hline Angulo & 333 & & & & \\
\hline \multicolumn{3}{|c|}{ PREDICHOS } & \multicolumn{2}{|c|}{ MEDIDOS } & DIFERENCIA \\
\hline \multicolumn{2}{|c|}{ Minimo } & 64.682755 & \multicolumn{2}{|c|}{59.8} & 4.882755 \\
\hline \multicolumn{2}{|c|}{ Maximo } & 74.904125 & \multicolumn{2}{|c|}{77.2} & -2.295875 \\
\hline \multicolumn{2}{|c|}{ Suma } & 3328.308854 & \multicolumn{2}{|c|}{3332.2} & -3.891146 \\
\hline \multicolumn{2}{|c|}{ Media } & 69.339768 & \multicolumn{2}{|c|}{69.420833} & -0.081065 \\
\hline \multicolumn{2}{|c|}{ Desviacion Estandar } & 2.14215 & \multicolumn{2}{|c|}{3.745551} & -1.603401 \\
\hline
\end{tabular}

TABLA 7. Modelo 3 Sector San Benito

\begin{tabular}{|c|c|c|c|c|c|}
\hline \multicolumn{2}{|c|}{ PROPIEDADES } & \multicolumn{2}{|c|}{ ESTADISTICOS } & & $\begin{array}{c}\text { RANGO DE } \\
\text { ERROR }\end{array}$ \\
\hline$x$ & 100 & \multicolumn{2}{|c|}{ Media } & -0.002 & -6.7871 \\
\hline$Y$ & 100 & \multicolumn{2}{|c|}{ RMS } & 3.786 & 9.6758 \\
\hline \multicolumn{6}{|l|}{ Angulo } \\
\hline \multicolumn{3}{|c|}{ PREDICHOS } & \multicolumn{2}{|c|}{ MEDIDOS } & DIFERENCIA \\
\hline \multicolumn{2}{|c|}{ Minimo } & 68.87119 & \multicolumn{2}{|c|}{66.4} & 2.47119 \\
\hline \multicolumn{2}{|c|}{ Maximo } & 80.946943 & \multicolumn{2}{|c|}{83.5} & -2.553057 \\
\hline \multicolumn{2}{|c|}{ Suma } & 3065.124209 & \multicolumn{2}{|c|}{3065.2} & -0.075791 \\
\hline \multicolumn{2}{|c|}{ Media } & 76.628105 & \multicolumn{2}{|c|}{76.63} & -0.001895 \\
\hline \multicolumn{2}{|c|}{ Desviacion Estandar } & 2.965103 & \multicolumn{2}{|c|}{4.335447} & -1.370344 \\
\hline
\end{tabular}

TABLA 8. Modelo 4 Sector San Benito

\begin{tabular}{|c|c|c|c|c|c|}
\hline \multicolumn{2}{|c|}{ PROPIEDADES } & \multicolumn{2}{|c|}{ ESTADISTICOS } & & $\begin{array}{c}\text { RANGO DE } \\
\text { ERROR }\end{array}$ \\
\hline $\mathrm{x}$ & 250 & \multicolumn{2}{|c|}{ Media } & -0.07 & -6.6649 \\
\hline Y & 250 & \multicolumn{2}{|c|}{ RMS } & 3.68 & 8.5662 \\
\hline \multicolumn{6}{|c|}{ Angulo } \\
\hline \multicolumn{3}{|c|}{ PREDICHOS } & \multicolumn{2}{|c|}{ MEDIDOS } & DIFERENCIA \\
\hline \multicolumn{2}{|c|}{ Minimo } & 70.164639 & \multicolumn{2}{|c|}{66.4} & 3.764639 \\
\hline \multicolumn{2}{|c|}{ Maximo } & 80.745971 & \multicolumn{2}{|c|}{83.5} & -2.754029 \\
\hline \multicolumn{2}{|c|}{ Suma } & 3062.414551 & \multicolumn{2}{|c|}{3065.2} & -2.785449 \\
\hline \multicolumn{2}{|c|}{ Media } & 76.560364 & \multicolumn{2}{|c|}{76.63} & -0.069636 \\
\hline \multicolumn{2}{|c|}{ Desviacion Estandar } & 2.603065 & \multicolumn{2}{|c|}{4.335447} & -1.732382 \\
\hline
\end{tabular}

\section{ANÁLISIS DE RESULTADOS}

Las configuraciones que presentaron menor error fueron las construidas mediante Kriging con los parámetros del modelo 2 (Laureles) y el modelo 1 (San Benito). Se evidencia una alta congruencia entre los datos estadísticos de los puntos predichos y los medidos, su media fue la más cercana a cero (0) y la desviación estándar fue la mínima entre todas las condiciones.

Aunque existe una buena correlación entre los estadísticos de los puntos medidos y los predichos, se encuentra una alta divergencia al comparar los valores de ruido registrados en campo y los que predice el método de interpolación. Estas diferencias se ubican entre el rango de $-6.42 \mathrm{~dB}(\mathrm{~A})$ y $7.56 \mathrm{~dB}(\mathrm{~A})$, lo cual es bastante significativo si se tiene en cuenta que el criterio de tolerancia usado internacionalmente para comparar los valores medidos con los simulados es \pm 3 $\mathrm{dB}$.

Por último, con relación a la norma colombiana, se analiza que el nivel mínimo registrado en las mediciones en ambos sectores supera los $65 \mathrm{~dB}(\mathrm{~A})$, siendo este el nivel máximo permitido en sectores de tranquilidad y ruido moderado (sector más restrictivo debido a zonas residenciales). Esto indica que todos los puntos exceden la norma, presentando además puntos críticos en las periferias correspondientes a vías principales.

\section{CONCLUSIONES}

En ambos escenarios, el método kriging se correlaciona de mejor manera con los estadísticos de los puntos medidos, la media general es más cercana a 0 y la desviación estándar es menor que en IDW, por lo cual es más preciso para generar curvas isoruido.

Los resultados obtenidos aplicando un mismo método de interpolación no presentan variaciones 
significativas. Sin embargo, al observar los mapas construidos con IDW se evidencia que no hay coherencia entre el comportamiento del ruido descrito por el modelo y el existente en la zona de estudio. Lo anterior se evidencia en variaciones de $5 \mathrm{~dB}(\mathrm{~A})$ entre puntos con características similares y que no se ajustan a la emisión generada por fuentes automotoras que son las que predominan en ambos sectores.

En el caso de los mapas obtenidos con kriging, se puede apreciar que los niveles más altos encontrados se deben a las vías principales e intercambios viales, lo que es congruente con la descripción dada anteriormente de los sectores. Sin embargo, se observa que para lograr una adecuada estimación se debe tomar un gran número de puntos de medición (rejilla de máximo $100 \mathrm{~m}$ entre vértices laureles, $60 \mathrm{~m}$ en San Benito), ya que la precisión de la estimación depende directamente del número de muestras obtenidas lo que se traduce en altos costos económicos.

Debido a que los métodos de interpolación no contemplan la manera como se propaga el sonido y la existencia de fenómenos acústicos como absorción, reflexión, difracción y atenuación generados por edificaciones y demás objetos existentes en el entorno, la representación de los niveles de presión sonora presenta grandes divergencias con relación al comportamiento del fenómeno in situ. Esta falencia se puede optimizar incrementando la resolución de la rejilla (mayor número de mediciones) con el fin de registrar las variaciones del sonido a medida que se propaga. Lo anterior se corrobora al analizar y comparar los niveles de ruido predichos con los puntos medidos.
Dado que los niveles de ruido ambiental exceden el umbral máximo permisible en ambos sectores, las autoridades ambientales deben adoptar mecanismos de control que propendan a la mitigación de los niveles de ruido con el fin de brindar espacios acústicamente sanos a la población.

\section{REFERENCIAS}

[1] Ministerio de Ambiente, Vivienda y Desarrollo Territorial. Res. 0627 Norma Nacional de ruido de emisión y ruido ambiental, 2006.

[2] E. Pueyo; J. Ortega \& D. Urraga. "Mapas de ruido nocturno en la ciudad". XVIII Congreso Internacional de Ingeniería Grafica. España, 2006.

[3] A. López; E. Quílez \& C. Abellán. "Mapas acústicos en zonas urbanas mediante predicción estadística". Proceedings $37^{\circ}$ Congreso Nacional de Acústica Tecniacústica, 2006.

[4] B. Bossam. "Noise Mapping In Urban Environments: Application at Suez City Center". Proceedings Computers \& Industrial Engineering CIE, pp. 1722-1727, 2009.

[5] V. Lobos. "Evaluación del ruido ambiental de la ciudad de Puerto Montt". Proceedings VI Congreso Iberoamericano de Acústica FIA, 2008.

[6] A. laaly-Sankari. "Urban noise mapping: the case of the city of El-Mina, North Lebanon". Proceedings Middle East \& North Africa Users Conference ESRI, 2010.

[7] S. Aditya; Y. Ghanshyam \& S. Biswas. "Traffic Noise Mapping". Gim International, Vol. 24, No. 6, 2010.

[8] Politécnico Colombiano Jaime Isaza Cadavid; Área Metropolitana Del Valle De Aburrá \& Universidad Nacional De Colombia - Sede Medellín. "Elaboración de los mapas acústicos y de concentraciones de monóxido de carbono para los municipios de la jurisdicción del Área Metropolitana del Valle de Aburrá”, Convenio 680 de 2005. Politécnico Colombiano Jaime Isaza Cadavid, 2006.

[9] R. Cañada et al. "Sistemas y análisis de la información geográfica”. Alfaomega, 2008. 